\title{
Introduction of optical tweezers in advanced physics laboratory
}

\section{Gang Wang}

Gang Wang, "Introduction of optical tweezers in advanced physics laboratory," Proc. SPIE 10452, 14th Conference on Education and Training in Optics and Photonics: ETOP 2017, 104521J (16 August 2017); doi: 10.1117/12.2269686

SDIE Event: 14th Conference on Education and Training in Optics and Photonics, ETOP 2017, 2017, Hangzhou, China 


\title{
Introduction of Optical tweezers in Advanced Physics Laboratory
}

\author{
Gang Wang \\ Department of Physics, Purdue University Fort Wayne, Fort Wayne, IN USA, 46805
}

\begin{abstract}
Laboratories are an essential part of undergraduate optoelectronics and photonics education. Of particular interest are the sequence of laboratories which offer students meaningful research experience within a reasonable time-frame limited by regular laboratory hours. We will present our introduction of optical tweezers into the upper-level physics laboratory. We developed the sequence of experiments in the Advanced Lab to offer students sufficient freedom to explore, rather than simply setting up a demonstration following certain recipes. We will also present its impact on our current curriculum of optoelectronics concentration within the physics program.
\end{abstract}

Keywords: Optoelectronics, photonics, optical tweezers, laboratory development, curriculum development, undergraduate education.

\section{INTRODUCTION}

Physics has been constantly changing with numerous renovations adding to the knowledgebase every year. On the contrary, curriculum of traditional undergraduate physics program has NOT undergoing the same amount of refreshing. From the contents to the order of physics curriculum were well developed decades ago and are very similar in many of the institutes around the world. ${ }^{[1-2]}$ This is especially noticeable in the area of optoelectronics and photonics, where the development of educational tools/methods are left behind the rapid growth of the research and technology in the field. Although it has been widely accepted that the undergraduate education are essential for the preparation of future work force, the bottle neck lies in finding a good approach to get undergraduate students exposed to the cutting edge science with limited time and background knowledge. This becomes particular challenging when laboratory courses are involved.

Physics is a science heavily based on the observations and experiments. To our best knowledge, there is no physics curriculum existing without a lab component. However, the purpose which a lab component serves has a wide variety. There was never lack of the debate among the relation between lectures and the laboratories in physics education. Many traditional laboratory investigations often fall into two contradictory categories: a replica of a demonstration of certain principle or a replica of a past research/investigation leading to the finding of a principle. Unfortunately, neither of the categories is satisfying for our optoelectronics and photonics education because the former category requires learning background knowledge before-hand and the latter category requires additional time outside delivery of concepts.

As an alternative and supplement to the traditional physics curriculum, we developed a sequence of course and laboratory investigation especially emphasizing optoelectronics and photonics education. This particular curriculum option is offered to under graduate students as the "Optoelectronics and photonics concentration" within the Purdue

14th Conference on Education and Training in Optics and Photonics: ETOP 2017, edited by Xu Liu,

Xi-Cheng Zhang, Proc. of SPIE Vol. 10452, 104521J - (o 2017 ICO, IEEE, OSA, SPIE

CCC code: $0277-786 X / 17 / \$ 18 \cdot$ doi: $10.1117 / 12.2269686$

Proc. of SPIE Vol. $10452104521 \mathrm{~J}-1$ 
University Fort Wayne (PFW) physics program. Moreover, the sequence is designed to allow a student to graduate in the regular timeframe, without extra burden for additional credit hours, and still gaining the necessary knowledge and skills to prepare them for their future careers in optoelectronics and photonics. Among the curriculum, a particular important component is the laboratory exploration. Our development of the laboratory focuses on the hands-on experience, conceptual understanding, (students') independent exploration, and professional communication. Different from the traditional procedure-based experimental investigates, our lab sequence (especially the upper-level labs) requires students to fully understand the topic and develop their own experimental procedure around a scheme determined at the instructor's choice. The general development of our adoption of such approach and the preliminary result of our pilot program were reported elsewhere ${ }^{[3]}$. We focus here on the introduction of optical trapping/manipulation of microscopic particles with optical tweezers into the undergraduate laboratory curriculum.

The momentum of light is commonly introduced into the undergraduate curriculum in their junior years. However, there are very little laboratory work associated with it. The students often leaves the classroom with impression that this is a modern topic: fancy, interesting, but that they will probably never see it or apply it again in their life. The difficulty of introducing momentum of light into laboratory lies on the constraint of cost of equipment and time of the investigation. We developed a sequence of optical tweezers laboratory so that students can construct a simple optical tweezer with He-Ne laser, optimize the system to trap and manipulate colloidal particles, and more importantly, investigate the physics associated with the colloidal particles. The total cost was very moderate: the project utilize mostly the current optical components from typical undergraduate optics labs (a $10 \mathrm{~mW}$ He-Ne laser, standard mirrors, lenses, and mechanics). The only additional requirement was a 100x microscope objective along with the colloids, costing approximately 200USD. We have implemented this project for 3 times with various revisions and additions. Our students were able to successfully reach the educational objective within the time limit of a 3 credit hour laboratory course.

\section{GENERAL LABORATORY STRUCTURE}

Recent physics educational research (PER) has found that interactive-engagement of students in the class significant improves students' conceptual understanding of material. ${ }^{[4-6]}$ It was often taken into grand that laboratory courses are automatically "interactive" because students are physically doing the experiments. In our practice, unfortunately, this is NOT always true. When students are given a well prescriptive procedure, they intend to focus more on following the recipe to reproduce or match the result with which their instructors expected. And a long term tracking data indicates they tend to forget their lab experience after an extended period even if they physically performed the activity. ${ }^{[7]}$ Instead, the purpose of the laboratory courses in our optoelectronics and photonics concentration are aiming at helping students to gain the skills to (1) identify the related concept for an unknown question or unfamiliar scenario, (2) to identify an appropriate tool/equipment needed for the investigation, (3) to develop an instrumentation plan and assembly the equipment accordingly (4) to carry out the measurement, and (5) to communicate their findings in a professional way.

These goals are difficult, if not impossible, to be finished in one semester or one course. Instead, we designed our laboratory courses in a layered structure. Starting from the freshman years, the general physics laboratories with the tradition contents of Mechanics, Electricity, Circuits, and Optics, our labs are carried out in a different approach. Throughout a semester, a sequence of investigations is selected to use identical instruments measuring certain physical quantities at various conditions. Students are not told what to do. Instead, they are asked to work on a related question before the lab starts (pre-labs). During the labs, they are only told what to study but not how. They need to determine what quantity, at what setup to measure, even though the instrument they are using is familiar to them. At the end of each lab period, they are given a final task. They need to perform and predict the result solely based on their investigation during the lab and reconcile any discrepancy (if there's any). The details of these labs has been reported 
earlier. ${ }^{[8-9]}$ These labs forced students into a habit of linking the observed phenomenon with the underline physics concepts. The traditional plug and chug mindset are not working in those labs. They need to understand what they were doing so that they could make a successful prediction for their final task. In our optical manipulation laboratory sequence, to prepare them for the understanding of the momentum of the light, as the first layer, we started sessions which are somewhat between the demonstration and investigation. We used a standard demonstration kit from W.M. Welch Scientific Company where four metal plates were attached onto a spinning pin in a vacuum tube. The plates spin when light incidents on them. In the first step of the general lab, we asked students to use different intensity of light to incident on the plates and set up a motion sensor to track the angular speed of the spinning of the plates. The students need to recognize the positive correlation between the light intensity and the spinning speed, which prepares them to understand the concept of the coupling of the momentum of light into material. We remark that this experiment by far is still the traditional "recipe" style of approach, where student solely following steps of setup and observe the result. It doesn't offer much independent thinking or investigation to explore and is AGAINST our laboratory philosophy. However, all these sessions are immediately followed by the tasks asking them to predict the light intensity needed to reach certain given speed of the spin. They are required to determine and adjust input light intensity so that the observed spinning angular speed matches with the instructor's request. Although the detailed spin dependency of the intensity relation is beyond a freshman/sophomore's understanding, the experiment force them to thinking into their data and explore the empirical formula of their experimental data. The students need to come up with their own model to explain the experimental observation. Given these first layer sessions often happens at their earlier years before they reach the advanced laboratory in their junior or senior years, it skips the complex and detailed theory behind optical momentum coupling. Instead, this experiment was solely serves as a step stone to prepare them with the direct experience in studying the momentum of light. This also positively build a good research habit for their future education.

In the second layer, when students reach the optical trapping laboratory for the advanced lab, students were introduced to all the available equipment with very limited information about experimental setup. They were required to perform literature research (with instructors supplied references) for the first two weeks to come up a plan for their experimental setup. They need to design and build an apparatus that would be able to trap micrometer-sized polystyrene beads and observe the behavior exhibited by them while trapped. The overarching goal was to build a reliable system and then, through the measurements of their motion to characterize any experimental parameters at their (students') choice, such as laser profile, liquid environment and so on.

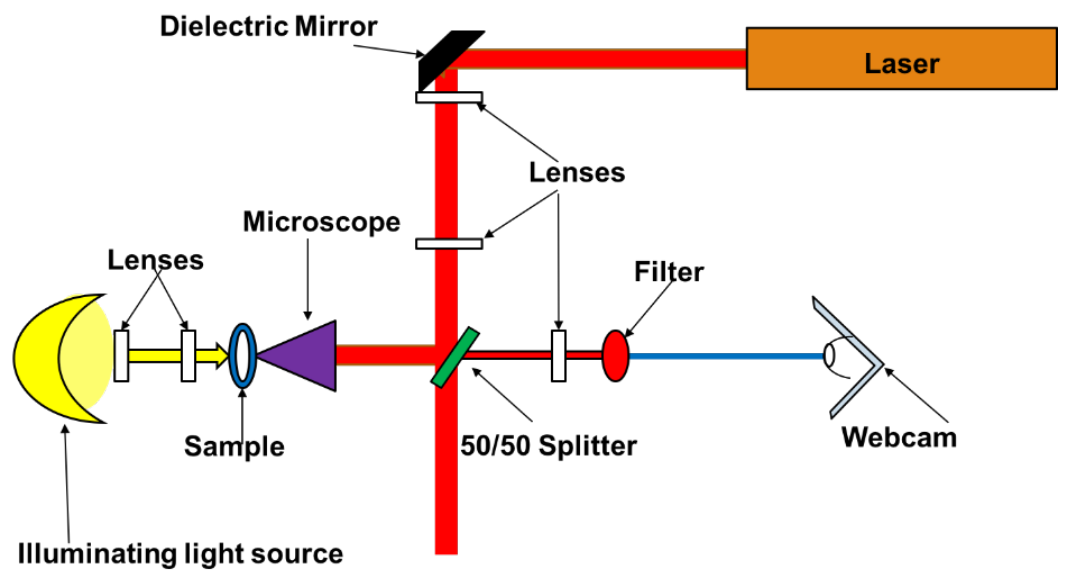

Figure 1: Schematic design of Sample student Experimental setup in Advanced Lab, fall semester 2016.

An example of such designs, by a student (Finn Buldt) from the Advanced Lab in the fall semester of 2016 was shown in Figure 1. The student used a JDS Uniphase $10 \mathrm{~mW}$ He-Ne laser, reflected by a few mirrors, collimated by lenses, 
and focused through an Olympus 100x microscope objective onto a sample cell made from two microscope cover glasses. The cover glasses were spaced by double tapes at a thickness approximately $80 \mu \mathrm{m}$ with colloidal liquid sealed in between. Some polystyrene spheres of $1.3 \mu \mathrm{m}$ (from Interactive dynamics) was suspended in the water or other liquid of students' choice. With proper support from instructor(s), average students can construct and tune their system to successfully trap a polystyrene sphere in the middle of the semester, at approximately $8^{\text {th }}$ week. During the instrumentation stage in the first half of the semester, students learn the basic skills of assembly and alignment of optics, safe operation of lasers, fundamental microscopy, machine shop skills, interfacing webcam and computers, processing video data, tracking and retrieving meaningful positional data of certain objects in the video. The instrumentation of the student was shown below, in figure 2.

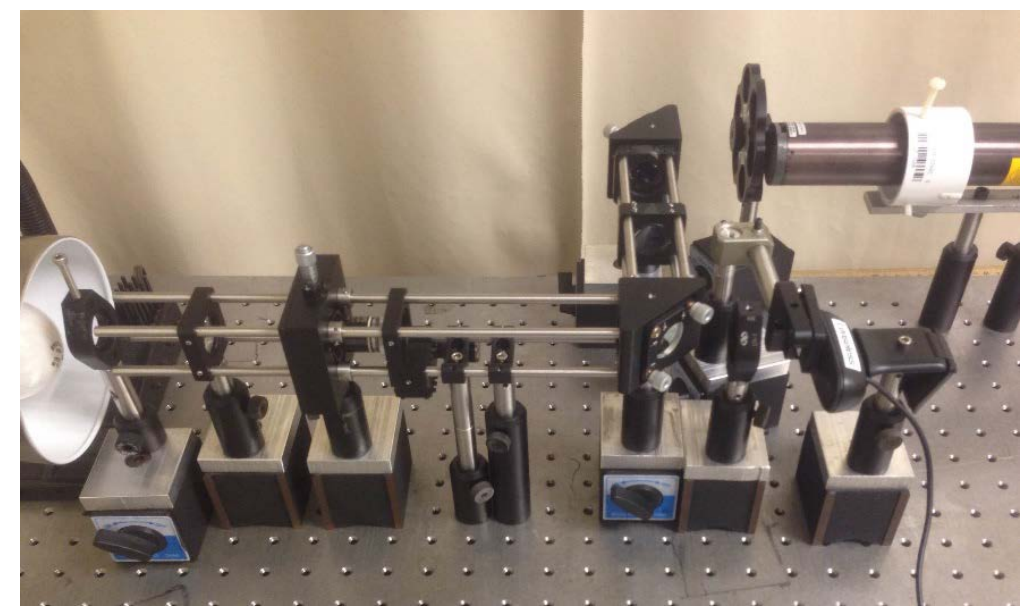

Figure 2: Sample of Experimental instrumentation by an Advanced Lab students, fall semester 2016.

At the next half of the semester, their preliminary result and understanding set the ground stone for the next investigation. They need to communicate their findings in poster/oral presentations with peer students and instructors. With all the feedback from instructor and their peers, they need to determine what to measure in the rest of the semester. Some common topics students used in the past years are:

- Measuring gravity coupled Brownian motion, i.e., students applying different trapping forces on the object, assuming the Brownian motion is isotropic, by measuring the anisotropic of the motion of the Brownian motion to characterize the gravity/buoyancy/density/ of the particle.

- Measuring the drag of the particle when they are trapped and manipulated by the laser beam to characterize the viscosity of the liquid/size of the particle. They had been experimenting with different liquids such as water, oil, ethanol alcohol, etc.

- Measuring the trapping strength to characterize the laser intensity profile/lenses aberration.

Some samples of the student presentations and experimental results are shown below in figure 3.

At this stage, the instructor kept the guidance to the minimal to offer students maximal freedom to explore the topics they are interested in. During the whole semester, the underlying physics concepts are NOT taught in the lecture. Instead, the students will explore and figure them out based on their own experiments. The advantage is two folded: (1) they learn physics concepts in the hands-on project, which helps understanding and long-term memorization. (2) they use the same time also learn the research method to explore unfamiliar territory. 

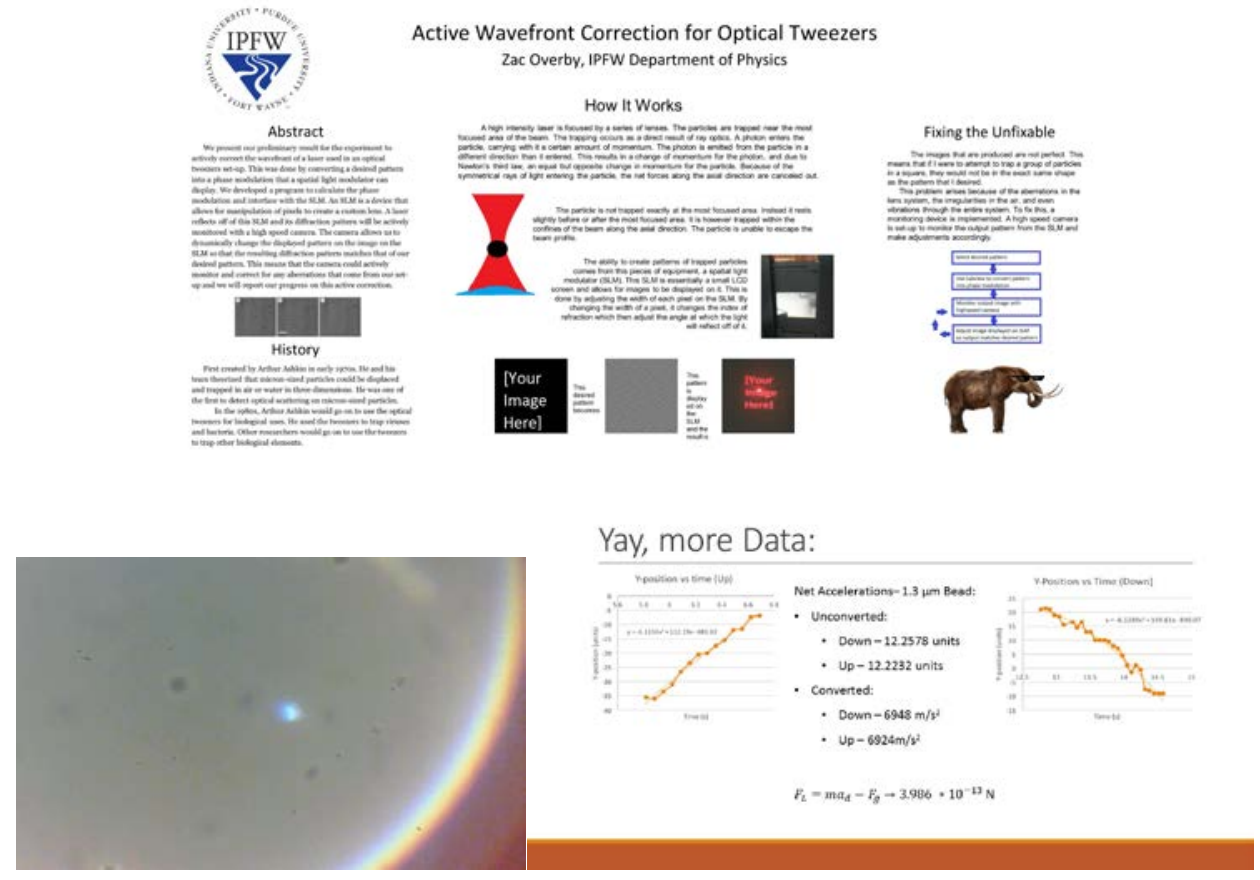

Figure 3: Sample Poster presentations and video screenshots from students in Modern physics lab and Advanced lab between 2015 - 2016.

As the final layer, after finishing all previous labs, students can move onto independent study or research experience with their capstone requirement - the senior thesis/research. Among all three layers, it is particularly important that the students gain hands-on experience and independence. The hands-on experience we refer here doesn't only mean they are using their hands to work on the project. It more refers to keep their minds on pondering through the project, a "mind-on" process so that students take the ownership of the project. The instructional hand-outs are often minimal, starting with a page at freshman level and reducing to only a few lines for juniors, only describing the theme and the overall goal of the experiments. The gradual withdrawal of instructor support leads students into a good work habit of critical thinking and independent investigation. When they reach the senior level, they should have learned the appropriate methodology for scientific research while they were learning the physical concepts. It's a more time efficient educational approach than the traditional laboratory courses.

As the report of their lab projects, they need to test their physical model with the final task at the first layer laboratories. They will communicate with peer students and instructors by poster presentation and oral presentations in the second layer laboratories. And in the third layer lab experience, they need to submit and cross-review their work in an online student-peer-reviewed journal, Journal of Advanced Undergraduate Physics Laboratory Investigations (JAUPLI) with reviewers and publishers from all participating colleges. We believe a consistent and coherent approach throughout the years of undergraduate education will better prepare our students with the curiosity toward unfamiliar phenomenon, the ability to visualize the link between concepts and phenomenon, the confidence in finding appropriate approach for investigation, and the necessary communication skills to report their findings. This allows more flexibility for our students in both continuing onto graduate school or into industrial working environment. 


\section{CONCLUSION: IMPACT ON UNDERGRADUATE EDUCATION}

Optoelectronics and photonics is one of the six tracks within the IPFW physics program. The traditional physics curriculum no longer fulfills the rapid progress of the knowledgebase in the field. At same time, the credit hours put an upper limit on the material and methodology we could adopt in our curriculum. We designed our new curriculum, utilizing a layer structured approach to combine the effective delivery of physics concepts and preparing students for the future career. This fits well in our overall educational framework, offering student opportunity to further explore their scientific interests and power them with skills to compete in a global technology environment. One particular example was the introductory of optical tweezers into undergraduate advanced lab. We designed a sequence of laboratories as a key pilot program to consistently provide students the hands-on experiences, which enhances their critical thinking and comprehensive conceptual understanding. Particularly important is the layered laboratory structure builds the scaffold for students to gain skills for independent research. The first two layers of laboratories has been systematically assessed and reported earlier. ${ }^{[3]}$ The pedagogic assessment of the third layer is under the ongoing process. The student feedback was positive. The optoelectronics and photonics track attracted the largest number of student enrollment in the department. We acknowledge that the assessment of the result is rather preliminary and yet to long term study. We will report the detailed assessment of the program elsewhere. ${ }^{[10]}$ Overall, our overarching laboratory principles and the coherent focus on curiosity, independence, and hands-on in our laboratory sequence resulted in our optoelectronics and photonics program being solid and practical.

\section{REFERENCES}

[1] Committee on Undergraduate Physics Education Research and Implementation, [Adapting to a Changing World-Challenges and Opportunities in Undergraduate Physics Education], the National Academies Press, Washington DC (2013).

[2] Committee on Education of the American Physical Society, [Guidelines for Undergraduate Physics Programs], AAPT, College Park, MD (1986).

[3] Wang, G. and Masters, M. "Optoelectronics and Photonics Laboratories at Indiana University Purdue University Fort Wayne", unpublished due to conference scheduling confliction.

[4] Meltzer, D.E. and Thornton, R.K., "Resource Letter ALIP--1: Active-Learning Instruction in Physics," Am. J. Phys. 80, 478 (2012).

[5] Hake, R.R., "Helping Students to Think Like Scientists in Socratic Dialogue-Inducing Labs," Phys. Teach. 50, 48 (2012).

[6] Hake, R. R. "Interactive-engagement versus traditional methods: A six-thousand-student survey of mechanics test data for introductory physics courses," American journal of Physics, 66, 64 (1998).

[7] Morgan, R.L., Whorton, J.E. and Gunsalus, C., "A comparison of short term and long term retention: Lecturecombined with discussion versus cooperative learning," Journal of Instructional Psychology, 27(1), 5358 (2000).

[8] Millspaw, J., Wang, G. and Masters, M.F., "Learning about light and optics in on-line general education classes using at-home experimentation," Proc. SPIE 9289, 92891M (2014).

[9] Grove, T. and Masters, MF., "Modifying the Optics Laboratory for Greater Conceptual Understanding," AAPT, (2009).

[10] Manuscript in preparation. 